\title{
CLASSIFICAÇÃo de CERÂMICAS ARQUEOLÓgICAS da BACIA AMAZÔNICA
}

Rose Mary Latini e Alfredo Victor Bellido Jr.

Departamento de Físico-Química, Instituto de Química, Universidade Federal Fluminense, Morro São João Batista s/n, 24020-150

Niterói - RJ

Marina Beatriz Agostini Vasconcellos

Divisão de Radioquímica, Instituto de Pesquisas Energéticas e Nucleares/CNEN, São Paulo - SP

Ondemar Ferreira Dias Junior

Departamento de História, Universidade Federal do Rio de Janeiro, Rio de Janeiro - RJ

Recebido em 30/5/00; aceito em 9/5/01

\begin{abstract}
CLASSIFICATION OF ARCHAEOLOGICAL CERAMICS FROM THE BRAZILIAN AMAZON BASIN. In order to elucidate the traditional classification of archaeological artefacts, a multielemental analytical method for characterisation of its micro and macro chemical constituents. combined with statistical multivariate analysis for classification, were used. Instrumental thermal neutron activation analysis, for elemental chemical determination, and three statistical methods: discriminant, cluster and modified cluster analysis were applied. The statistical results obtained for the samples from Iquiri, Quinari and Xapuri archaeological phases were in good agreement with the conventional archaeological classification. Iaco and Jacuru archaeological phase were not characterised as homogenous groups. Iquiri phase were the most distinct in relation to the other analysed groups. An homogeneous group for 54\% collected samples at the Los Angeles site was also found, this could be characterised as a new archaeological phase.
\end{abstract}

Keywords: archaeometry; ancient ceramics; neutron activation analysis.

\section{INTRODUÇÃO}

A identificação e classificação de cerâmicas a partir de uma análise química multielementar em combinação com análise estatística de multivariáveis pode ser utilizada para determinação de procedência de cerâmicas, matéria-prima (argila) e técnicas de fabricação. Estes dados podem ajudar, por exemplo, no esclarecimento de intercâmbio cultural e comercial entre comunidades antigas. Este trabalho tem por objetivo contribuir com uma base analítica no estudo de classificação arqueológica, a qual poderá ser utilizada como referência em trabalhos posteriores para um melhor entendimento das culturas pré-históricas na Bacia Amazônica.

As cerâmicas, por serem extremamente resistentes às condições do tempo e do meio circundante, são objetos de grande valor arqueológico ${ }^{1}$. Na confecção destas, em muitos casos as argilas são misturadas com "temperos", formando uma pasta para fabricação das mesmas. Como temperos são normalmente utilizados material orgânico, como cascas de árvores, areia, cacos cerâmicos triturados e conchas entre outros. Elas são moldadas no formato desejado a partir da pasta úmida que depois é endurecida pelo cozimento em forno.

Nos grupos de argilominerais, as espécies mineralógicas e a respectiva composição geoquímica das argilas, apresentam variações causadas pelas diferentes condições geológicas de formação e em função do grau de substituições isomórficas ou troca por similaridade e cátions trocáveis. A importância dos elementos traços na composição de cerâmicas reside no fato de que estas refletem as características de composição química original das argilas que a constituem. Considera-se estatisticamente improvável que a concentração de vários elementos traços de argilas de uma determinada localidade coincida

*e-mail: rmlatini@domain.com.br quantitativamente com a de argilas de outras localidades (procedências) $)^{2}$.

Para caracterização do material arqueológico foi utilizado o método de Análise por Ativação Neutrônica (AAN) que é uma técnica analítica qualitativa, quantitativa e multielementar, a qual envolve dois processos: irradiação das amostras em um reator nuclear e medida da atividade induzida por espectroscopia gama. A irradiação ou ativação é realizada através da exposição da amostra à um fluxo de nêutrons, transformando os elementos estáveis em radioativos pela reação nuclear $(n, \gamma)$. A medida da radioatividade é realizada em um detector semicondutor de alta resolução ${ }^{3}$. Este método é muito bem estabelecido, preciso e apresenta boa sensibilidade para um grande número de elementos químicos, desde concentrações em $\%$, até níveis de traços (limites de detecção abaixo de $p p b$ para muitos deles) ${ }^{4}$. Assim, a AAN permite caracterizar cada um dos fragmentos cerâmicos atribuindo-lhe uma identidade (impressão digital) ${ }^{5}$ com base na sua composição química, tendo se mostrado muito útil na caracterização de objetos relacionados entre si permitindo, em muitas ocasiões, que estes sejam relacionados a uma origem comum (procedência) ${ }^{6,7}$.

Para a classificação das cerâmicas foi necessário a utilização de técnicas estatísticas avançadas (análise estatística de multivariáveis), devido ao grande número de elementos e amostras analisados. As técnicas tiveram por objetivo identificar agrupamentos em função de suas similaridades, levando a préclassificação arqueológica feita a partir de macrodiferenciações para uma classificação a nível ordenado de composição química elementar.

\section{As cerâmicas}

Os vasos inteiros ou fragmentos cerâmicos encontrados são pré-classificados e catalogados pelos arqueólogos em função de atributos tais como: nível de soterramento do material en- 
contrado, cor, forma do vaso, textura do material, tempero utilizado entre outros. Estes parâmetros podem revelar tradições e interações culturais entre comunidades antigas e, quando estas interagem mais intensamente entre si podem representar uma fase arqueológica. Uma tradição cultural pode ser entendida como fases arqueológicas que compartilham de um mesmo conjunto de técnicas diagnosticadas pelos atributos acima citados ${ }^{8}$.

A área de estudo situa-se na Bacia Hidrográfica do Rio Purus, na região do Estado do Acre, localizado na Amazônia Ocidental à sudeste do Estado do Amazonas; faz divisa com os Estados do Amazonas e Rondônia e fronteira com Peru e Bolívia. Historicamente, com os resultados até agora disponíveis provenientes do Instituto de Arqueologia Brasileira (IAB/ PRONAPABA), a partir de 1977, acredita-se existir neste Estado duas Tradições Culturais principais, uma sobre a Bacia do Rio Juruá e outra sobre a Bacia do Rio Purus, a qual é abordada neste trabalho. Uma característica de alguns dos sítios encontrados é que estes possuem ao seu redor uma estrutura de terra circular em forma de valeta, com acúmulo de terra em forma de mureta na área externa do círculo. Esse tipo de estrutura já havia sido noticiada em outras partes da Amazônia ${ }^{9}$ e a cerâmica encontrada nas mesmas comprova a sua antiguidade e a sua relação com a pré-história local ${ }^{10}$. Recentemente, foram encontradas na região outras estruturas de terra com diferentes formas geométricas ${ }^{11}$.

As cerâmicas encontradas nos sítios arqueológicos em estudo (Figura 1), foram pré-classificadas pelos arqueólogos Dias e Carvalho ${ }^{12}$ como pertencente a Tradição Cultural denominada Quinari, a qual até o momento é composta por cinco Fases Arqueológicas: Quinari, Iaco, Xapuri, Jacuru e Iquiri. Porém, as amostras provenientes do Sítio Arqueológico Los Angeles ainda não possuem fase arqueológica definida.

\section{PARTE EXPERIMENTAL}

De um total de 162 fragmentos cerâmicos analisados, 20 pertencem à fase arqueológica Quinari, 18 são da fase Iquiri, 22 fragmentos da fase arqueológica Iaco, 11 da fase Jacuru, 17 da fase Xapuri e 74 fragmentos cerâmicos do Sítio Arqueológico Los Angeles (SALA) com fase a ser designada.

A AAN foi o método utilizado para identificação e caracterização do material arqueológico. Neste trabalho fez-se uso do método de comparação, o qual consiste em utilizar um padrão de referência de composição química semelhante à amostra analisada. A concentração dos elementos na amostra são obtidas diretamente pela comparação com a quantidade conhecida dos mesmos elementos presentes no padrão. $\mathrm{O}$ uso de padrão elimina a necessidade do conhecimento exato de dados nucleares do nuclídeo alvo e do produto de reação. O padrão de referência utilizado foi o Podmore Red Clay; elaborado na Universidade de Manchester, Inglaterra e certificado através de intercomparação em laboratórios de referência ${ }^{13}$.

Inicialmente foram retirados aproximadamente 2 milímetros da superfície de cada um dos fragmentos cerâmicos com uma broca de diamante para remoção de possíveis contaminantes. Após esta etapa, as amostras foram pulverizadas em gral de ágata, homogeinizadas e secas por 12 horas a $80{ }^{\circ} \mathrm{C}$. Aproximadamente $200 \mathrm{mg}( \pm 20 \mathrm{mg})$ de cerâmicas e padrões foram acuradamente pesados em cápsulas de polietileno. Procurou-se manter, aproximadamente, a mesma massa entre amostras e

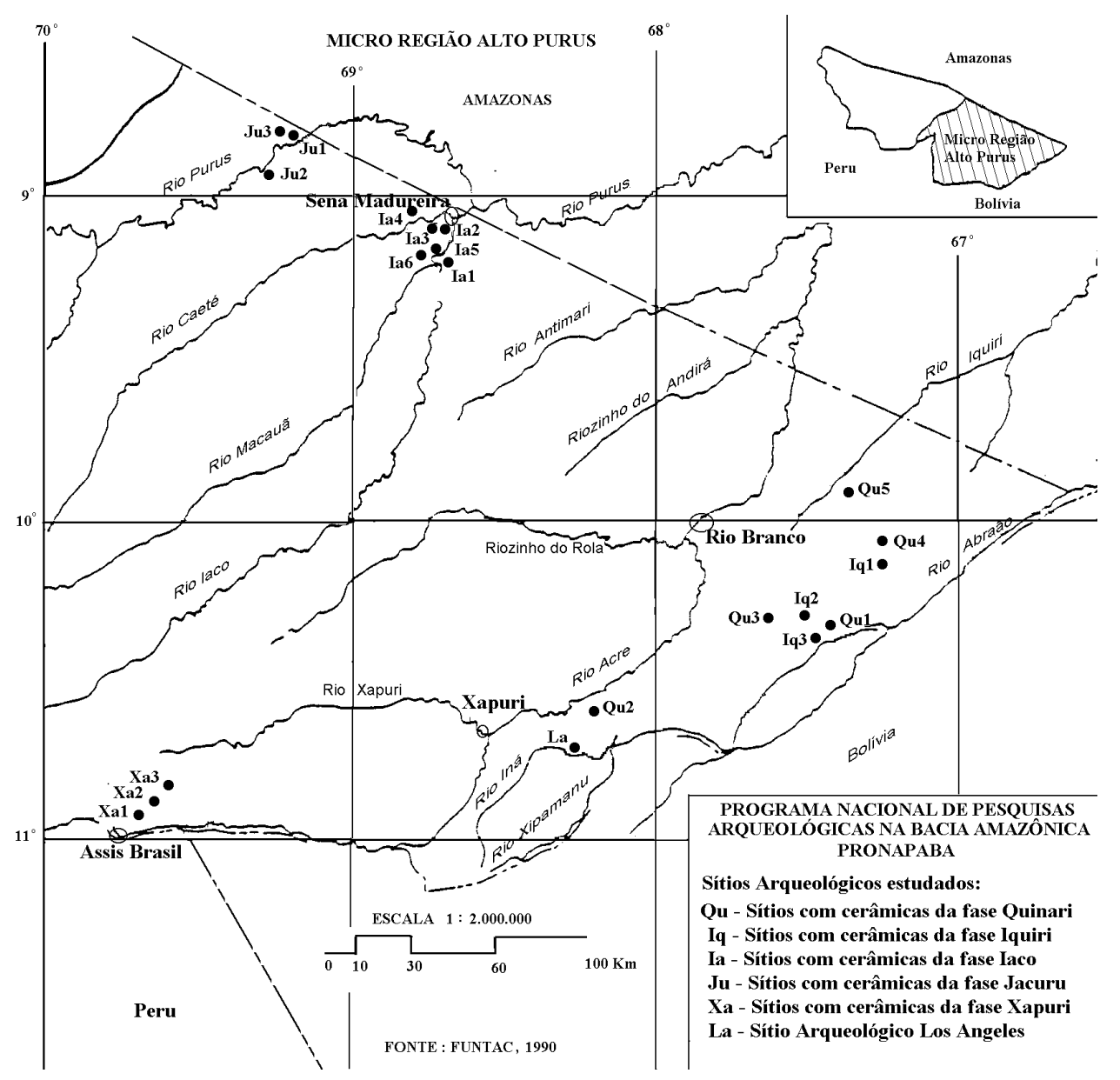

Figura 1. Mapa de localização dos Sítios Arqueológicos em estudo, modificado do PRONAPABA 
padrões com a finalidade de garantir as mesmas geometrias. Em seguida, as cápsulas foram apropriadamente seladas. Em todas as etapas do trabalho manteve-se as mesmas condições de irradiação e contagem para amostras e padrões, os quais foram submetidos a um fluxo de nêutrons térmicos da ordem de $10^{12} \mathrm{n} \cdot \mathrm{cm}^{-2} \cdot \mathrm{s}^{-1}$ do reator de pesquisas IEA-R1 do Instituto de Pesquisas Energéticas e Nucleares - IPEN/CNEN.

A ativação das amostras foi realizada em duas etapas, de acordo com a meia-vida dos radionuclídeos medidos. Nas irradiações de curta duração utilizou-se um sistema pneumático para lançar as amostras, pois o tempo de irradiação foi de apenas 1,5 min. Estas amostras foram contadas por $4 \mathrm{~min}$. após um tempo de decaimento de $10 \mathrm{~min}$. Para análise de radionuclídeos de meia-vida longa as amostras foram irradiadas por 8 horas e contadas por 2 horas após um tempo de decaimento de 15 dias.

A detecção da radiação gama oriunda das amostras ativadas foi feita por meio de detector HPGe cuja resolução é de 1,83 $\mathrm{keV}$ para o fotopico de energia de $1332 \mathrm{keV}$ do ${ }^{60} \mathrm{Co}$. A eletrônica associada consistiu em um amplificador e um analisador multicanal de 4096 canais. A aquisição dos espectros foi feita utilizando-se o programa RAE ${ }^{14}$. Para análise dos espectros e cálculo das concentrações dos elementos químicos utilizou-se o programa GRGAN ${ }^{15}$, considerando apenas os raios gamas livres de interferentes ${ }^{15}$.

Todas as amostras foram analisadas em duplicatas e algumas em triplicatas (no caso de não se ter obtido boa reprodutibilidade). Para cada uma delas foram determinados 20 elementos químicos: Al, Ce, Co, Cr, Cs, Dy, Eu, Fe, Hf, Lu, $\mathrm{Mn}, \mathrm{Na}, \mathrm{Rb}, \mathrm{Sb}, \mathrm{Sc}, \mathrm{Sm}$, Ta, Th, Ti, e V. As cápsulas de polietileno foram também analisadas para identificação de possíveis impurezas, não tendo sido encontrada nenhuma interferência significante. Os radionuclídeos e os respectivos raios $\gamma$ medidos podem ser vistos na Tabela 1 .

\section{ANÁLISE ESTATÍSTICA DE MULTIVARIÁVEIS}

As técnicas de análise estatística de multivariáveis tem por finalidade a classificação e ordenação de um conjunto de dados. As técnicas de classificação são destinadas a produzir grupos ou conglomerados de objetos (amostras) semelhantes, segundo suas características (variáveis) e, as de ordenação têm por finalidade o estudo das correlações existente entre as variáveis de um grupo de objetos, não impedindo também de serem utilizadas para classificação ${ }^{16}$.
O propósito é considerar diversas variáveis simultaneamente, sendo todas de igual importância no início da análise, para tal é necessário uma análise exploratória dos dados. Uma consideração que normalmente é feita é de que os dados seguem distribuição normal. De forma geral, se todas as variáveis estudadas seguem uma distribuição normal individualmente, a distribuição resultante também o será ${ }^{17}$. Como as variáveis foram apresentadas em diferentes unidades, foi realizada uma padronização dos dados para que as mesmas pudessem ser comparadas dentro de uma mesma ordem de grandeza. O método utilizado foi a transformação "z-scores" que gerou novas variáveis com média zero e desvio padrão igual a um. Sendo assim, a concentração de cada elemento passou a ter um valor em unidades de desvio padrão dado pela equação:

$$
z_{s i}=\frac{X s i-\bar{X} i}{\sigma_{i}}
$$

onde:

$Z_{s i}=$ desvio padrão do elemento i na amostra s.

$\underline{X} s i=$ concentração do elemento i na amostra s.

$\bar{X} i$ e $\sigma_{i}=$ são a média e o desvio padrão determinados para o elemento em toda a população.

Nos métodos de classificação, as medidas de distância empregadas quantificam o grau de associação entre um par de amostras ou grupos em função de suas variáveis. Quanto mais próximas forem duas amostras, menor a distância entre os pontos representativos dessas amostras num espaço n-dimensional, maior então a similaridade entre elas. Um índice de distância corresponde então a uma dissimilaridade ${ }^{18}$. A distância Euclideana foi utilizada para medir a distância entre amostras individuais e a distância de Mahalanobis empregada para se calcular distâncias entre agrupamentos de amostras.

As técnicas de classificação empregadas foram a análise de discriminantes e a análise de conglomerados. O objetivo da análise de discriminantes foi de interpretar grupos de amostras pré-classificadas pelos arqueólogos, com base nas medidas de suas variáveis ${ }^{19}$. Esta técnica não consiste em estabelecer grupos, já que estes são a priori estabelecidos, mas interpretá-los a partir do conjunto de variáveis medidas para cada amostra produzindo assim, uma função discriminante entre os grupos.

No método de conglomerados não é necessário considerar nenhuma classificação inicial para os grupos, pois o método é

Tabela 1. Elementos e respectivos radionuclídeos analisados (a-anos, d-dias, h-horas, C-curta e L-longa)

\begin{tabular}{|c|c|c|c|c|}
\hline Elemento & Radionuclídeo & Meia-vida & Energia $\gamma[\mathrm{keV}]$ & Tipo de Irradiação \\
\hline $\mathrm{Na}$ & $\mathrm{Na}-24$ & $15,03 \mathrm{~h}$ & 1368,5 & $\mathrm{C}$ \\
\hline $\mathrm{Al}$ & Al-28 & $2,25 \mathrm{~m}$ & 1778,8 & $\mathrm{C}$ \\
\hline $\mathrm{Ti}$ & Ti-51 & $5,79 \mathrm{~m}$ & 320,1 & $\mathrm{C}$ \\
\hline $\mathrm{V}$ & V-52 & $3,75 \mathrm{~m}$ & 1434,1 & $\mathrm{C}$ \\
\hline $\mathrm{Mn}$ & Mn-56 & $2,58 \mathrm{~h}$ & 1811,0 & $\mathrm{C}$ \\
\hline Dy & Dy-165 & $2,33 \mathrm{~h}$ & 94,6 & $\mathrm{C}$ \\
\hline $\mathrm{Sc}$ & $\mathrm{Sc}-46$ & $83,9 \mathrm{~d}$ & 889,3 & $\mathrm{~L}$ \\
\hline $\mathrm{Fe}$ & $\mathrm{Fe}-59$ & $44,6 \mathrm{~d}$ & 1099,3 & $\mathrm{~L}$ \\
\hline $\mathrm{Co}$ & Co-60 & 5,28 a & 1333,0 & $\mathrm{~L}$ \\
\hline $\mathrm{Rb}$ & $\mathrm{Rb}-86$ & $18,82 \mathrm{~d}$ & 1076,6 & $\mathrm{~L}$ \\
\hline $\mathrm{Sb}$ & $\mathrm{Sb}-124$ & $60,2 \mathrm{~d}$ & 602,7 & $\mathrm{~L}$ \\
\hline $\mathrm{Cs}$ & Cs-134 & $2,04 \mathrm{a}$ & 795,8 & $\mathrm{~L}$ \\
\hline $\mathrm{Ce}$ & $\mathrm{Ce}-141$ & $32,5 \mathrm{~d}$ & 145,5 & $\mathrm{~L}$ \\
\hline $\mathrm{Sm}$ & Sm-153 & $46,7 \mathrm{~h}$ & 103,2 & $\mathrm{~L}$ \\
\hline $\mathrm{Lu}$ & Lu-177 & $6,74 \mathrm{~d}$ & 208,3 & $\mathrm{~L}$ \\
\hline Hf & Hf-181 & $42,5 \mathrm{~d}$ & 482,1 & $\mathrm{~L}$ \\
\hline $\mathrm{Ta}$ & Тa-182 & $115,0 \mathrm{~d}$ & 1221,4 & $\mathrm{~L}$ \\
\hline Th & $\mathrm{Pa}-233$ & $27,4 \mathrm{~d}$ & 311,9 & $\mathrm{~L}$ \\
\hline
\end{tabular}


usado para classificar as amostras de acordo com suas dissimilaridades de composição química (variáveis) no espaço ndimensional. $\mathrm{O}$ resultado da análise de conglomerados frequentemente é apresentada na forma de dendograma, que são gráficos bidimensionais de fácil visualização. Para formação dos agrupamentos, diferentes critérios de aglomeração podem ser utilizados. O método Ward ou método de variância mínima foi considerado o procedimento mais eficiente para formação destes grupos, uma vez que um objeto só será incorporado à um grupo se essa reunião proporcionar a menor variância no sistema ${ }^{18}$.

$\mathrm{O}$ efeito de correlação deve ser investigado em estudos de classificação, já que a análise de conglomerados trata os dados como sendo variáveis independentes e não correlacionadas. As causas mais comuns são correlações naturais devido à geoquímica dos elementos presentes ou correlações provocadas durante a confecção das cerâmicas quando há adição de tempero. Outro efeito a ser investigado é o de diluição, pois os valores da composição química elementar podem vir a ser diluídos pela adição de "temperos" 20 , como por exemplo: areia ou pedaços de conchas que podem ser usados em diferentes quantidades no mesmo tipo de argila mudando assim a concentração dos elementos (diluição) na cerâmica confeccionada.

\section{RESULTADOS E DISCUSSÕES}

\section{Tratamento Estatístico dos Dados Analíticos}

Como variáveis foram utilizadas as concentrações dos 20 elementos químicos obtidos pela AAN para as 162 amostras analisadas. Testes de distribuição de frequência foram realizados nesta matriz de dados verificando-se que os elementos seguem individualmente distribuição normal.

O pacote estatístico utilizado para a análise de multivariáveis foi o Statistical Analysis System (SAS); sendo que outros pacotes estatísticos também podem produzir os mesmos resultados, porém com visualização de dados de entrada e de saída diferenciados.

Os métodos de classificação foram inicialmente utilizados com a finalidade de se testar diferentes hipóteses: a consistência da pré-classificação arqueológica (análise de discriminante) e a classificação puramente a partir da composição química (análise de conglomerados).

\section{Análise de Discriminantes}

Inicialmente foi dado como entrada os 162 fragmentos cerâmicos como pertencente a um dos 6 grupos (fases arqueológicas) pré-definidos pelos arqueológos. A medida de distância utilizada foi a de Mahalanobis ${ }^{21}$.

Um total de 63 amostras foram deslocadas pela função discriminante para um dos outros grupos pré-definidos. As amostras da Fase Iaco foram as que mais se dispersaram entre os outros grupos (15 amostras). De cada um dos grupos formados pelo discriminantes foram separadas as amostras que tinham valores das variáveis fora do intervalo de confiança de 95\% ("outliers"). Desta maneira, as amostras restantes são as mais representativas de cada um dos grupos.
Foram poucas as amostras representativas das Fases Iaco e Jacuru, mostrando que as amostras de cada um dos grupos não possuem homogeneidade entre elas. Já as Fases Iquiri e Xapuri possuem mais de $80 \%$ de amostras representativas sendo considerados os grupos mais homogêneos. $\mathrm{O}$ inconveniente do método de discriminantes portanto, é que ele não consegue prever a existência de outros grupos, uma vez que cria uma função discriminante para cada um dos grupos inicialmente definidos. A Tabela 2 apresenta um resumo dos resultados obtidos pela análise de discriminantes e a Figura 2 ilustra esses resultados em um gráfico bi-dimensional.

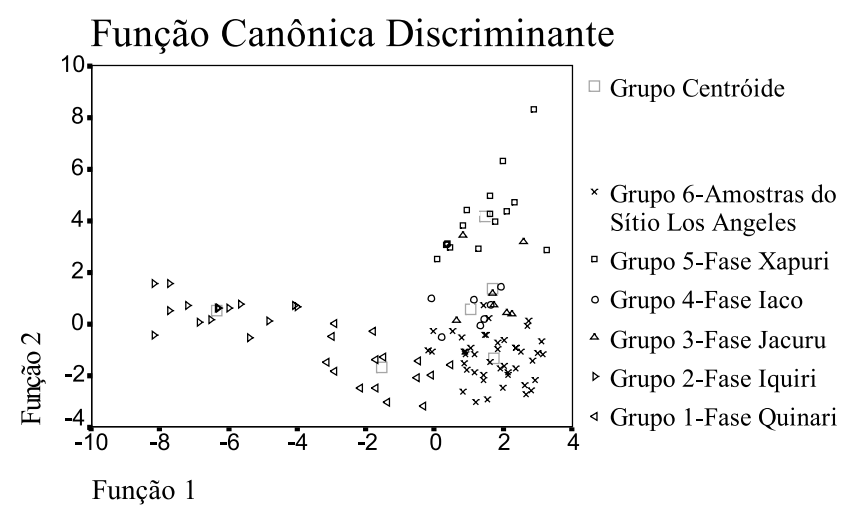

Figura 2. Resultado da Análise de Discriminantes

\section{Método de Conglomerados}

A dstância Euclideana quadrada foi usada como medida de distância e a técnica hierárquica de conglomerados foi utilizada na subrotina Ward (variância mínima).

Para facilitar a visualização da metodologia utilizada na análise de conglomerados, um diagrama de blocos foi elaborado para mostrar a formação dos grupos passo a passo. Os 162 fragmentos cerâmicos foram divididos em dois grupos em função de suas dissimilaridades, um contendo 128 amostras e o outro 34, este último por sua vez foi dividido em 3 subgrupos, contendo algumas das amostras das fases arqueológicas Iquiri, Iaco e Quinari, conforme pode ser observado na Figura 3. A seguir, aplicou-se novamente o método para o grupo contendo 128 amostras. Como resultado desta metodologia obteve-se um total de 12 sub-grupos. A Figura 4 ilustra um dos dendogramas obtidos, contendo os sub-grupos SB1, SB2 e SB3.

A existência do efeito correlações internas foi verificado pelo método de Pearson para identificação das variáveis que se apresentavam correlacionadas. Com o intuito de minimizar este efeito nos resultados obtidos na análise de conglomerados foram feitas duas tentativas: a primeira foi retirar uma das variáveis correlacionadas, ocasionando assim uma redução no número de variáveis da matriz de dados de 20 para 10 . A segunda, foi realizada utilizando-se o método de correção de correlações proposto por Bellido et al. ${ }^{13}$, método modificado de conglomerados, o qual utiliza todas as variáveis e quebra as correlações

Tabela 2. Resumo da Análise de Discriminantes

\begin{tabular}{lccccc}
\hline Nome do Grupo & $\begin{array}{c}\text { Amostras } \\
\text { Iniciais }\end{array}$ & $\begin{array}{c}\text { Resultado } \\
\text { do Discriminante }\end{array}$ & $\begin{array}{c}\text { Concordância } \\
(\%)\end{array}$ & $\begin{array}{c}\text { Mais repre- } \\
\text { sentativas }\end{array}$ & $\begin{array}{c}\text { Deslocamento } \\
\text { para }\end{array}$ \\
\hline Iquiri (Iq) & 18 & 15 & 83 & 11 & Qu \\
Quinari (Qu) & 20 & 15 & 75 & 13 & $\mathrm{Iq} \mathrm{e} \mathrm{La}$ \\
Iaco (Ia) & 22 & 7 & 32 & 4 & $\mathrm{Qu}, \mathrm{Ja}, \mathrm{Xa} \mathrm{e} \mathrm{La}$ \\
Xapuri (Xa) & 17 & 15 & 88 & 10 & $\mathrm{Ia}$ \\
Jacuru (Ja) & 11 & 7 & 64 & 3 & $\mathrm{Ia}$ e Xa \\
SALA (La) & 74 & 40 & 54 & 29 & $\mathrm{Qu}, \mathrm{Ja}, \mathrm{Xa}$ e Ia \\
\hline
\end{tabular}




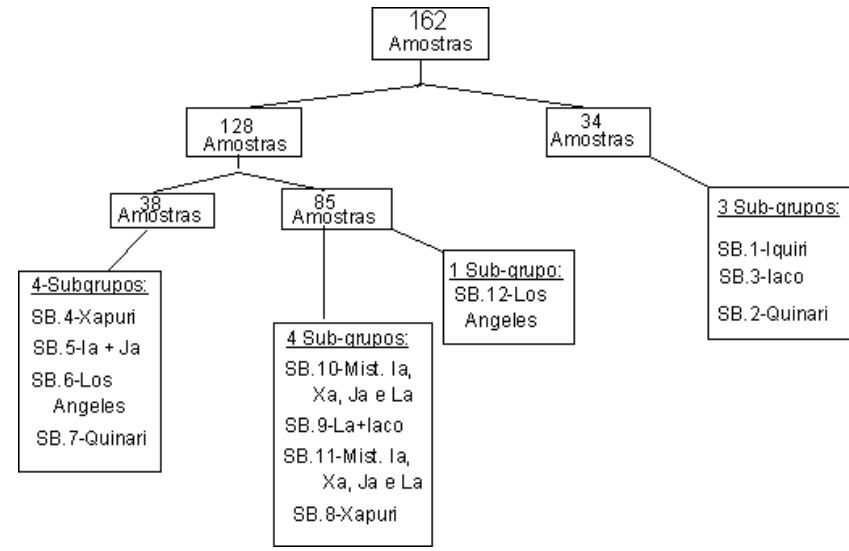

Figura 3. Diagrama de blocos da formação de grupos pela análise de conglomerados

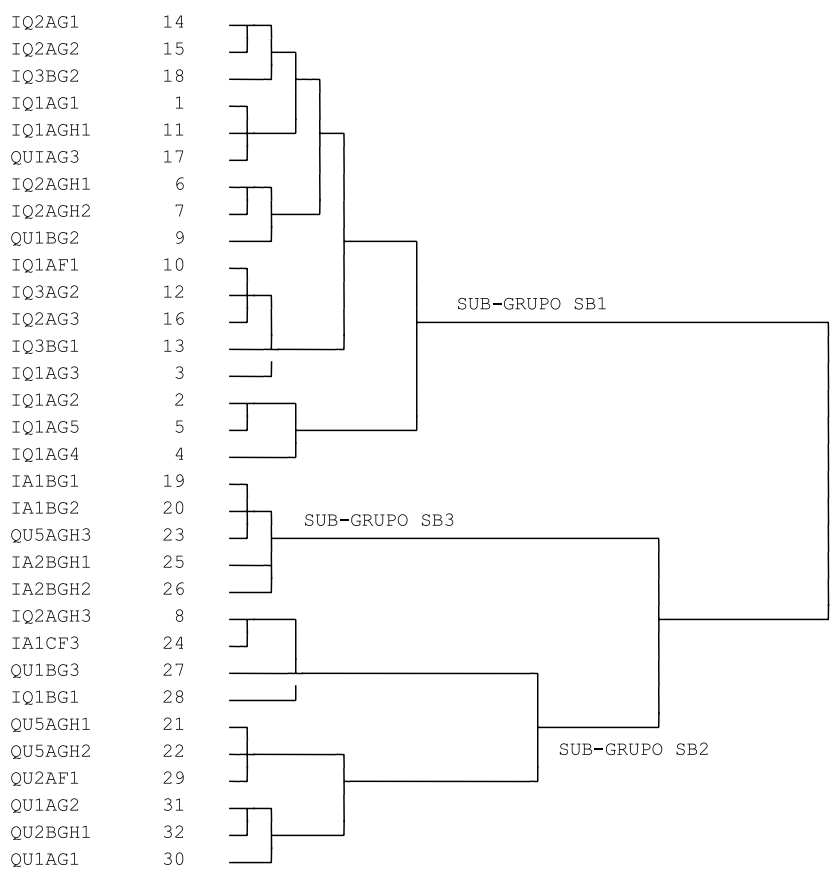

Figura 4. Dendograma mostrando amostras dos sub-grupos SB.1, $S B .3$ e SB.2

existentes a partir do emprego da análise de componentes principais para determinação da direção ou sentido da correlação ${ }^{13}$ Não foi verificado nenhuma alteração entre os grupos formados pelo método modificado de conglomerados e os formados pelo método de conglomerados convencional sem as variáveis correlacionadas.

Cada um dos sub-grupos resultantes do método de conglomerados foram separadamente estudados para verificação do efeito de diluição, o qual pode ser causado pela adição de materiais diluentes utilizados como temperos. Para se verificar este efeito foi utilizado o fator de diluição, $f_{i}$, proposto por Mommsen et al. ${ }^{20}$. Um grupo está diluído em relação à outro quando o fator de diluição para cada um dos elementos medidos é aproximadamente o mesmo e diferente da unidade. Quando porém, $f_{i}$, é aproximadamente igual à unidade nenhuma diluição está presente. Grupos com composição diferente possuem valores dispersos de $f_{i}$. .

A partir dos estudos realizados, as amostras estudadas foram então classificadas em função puramente da sua composição química nos seguintes grupos:

\section{- Grupo A}

Constituído basicamente de amostras da fase Iquiri (subgrupo 1); contém duas amostras da fase Quinari, as quais passam a ser reclassificadas como sendo pertencente à este grupo.

\section{- Grupo B}

Constituído basicamente de amostras da fase Quinari; o fator de diluição médio mostra que o sub-grupo 2 confeccionou as cerâmicas utilizando-se da mesma argila que o sub-grupo 7, porém acrescentando "tempero" em quantidades diferentes. Além das amostras da fase Quinari o grupo contém 2 amostras da fase Iquiri e 1 amostra da fase Iaco que passam então a ser assim reclassificadas em função da composição química.

\section{- Grupo C}

Constituído de uma mistura de amostras das fases Iaco e Jacuru, representado pela junção dos sub-grupos 3 e 5 .

\section{- Grupo D}

Constituído pelas amostras da fase Xapuri, representado pela junção dos sub-grupos 4 e 8 .

\section{- Grupo E}

Constituído por amostras do Sítio Arqueológico Los Angeles, contendo as amostras dos sub-grupos 6 e 12 e uma amostra da fase Jacuru que passa a ser assim reclassificada.

\section{- Grupo F}

Constituído das amostras dos sub-grupos 9, 10 e 11, os quais são formados por uma mistura de amostras do Sítio Los Angeles, fase Iaco, fase Xapuri e fase Jacuru. Grupo bastante semelhante ao Grupo E, sendo diferenciado principalmente pelos elementos $\mathrm{Na}$, Co, Eu e Dy.

A caracterização de cada um dos grupos definidos acima foi feita pela média e desvio padrão de cada um dos elementos estudados (ver Tabela 3).

\section{CONCLUSÕES}

Com os resultados da análise estatística de multivariáveis pode-se verificar que em relação à hipótese da validade da préclassificação das cerâmicas feita pelos arqueólogos, a análise de discriminante mostrou uma concordância, em média, de $70 \%$ entre os resultados obtidos a partir da composição química e a pré-classificação arqueológica. As fases arqueológicas Xapuri, Iquiri e Quinari foram as que apresentaram maior concordância e as fases Iaco e Jacuru, as que menos se definiram como um grupo homogêneo. No que diz respeito às amostras coletadas no Sítio Arqueológico Los Angeles, com fase ainda a ser designada pelos arqueólogos, o discriminante mostrou que 54\% de suas amostras são integrantes de um grupo homogêneo, as quais poderiam caracterizar uma outra fase arqueológica. Das funções discriminantes traçadas, para cada um dos grupos, pode-se verificar que as amostras integrantes da fase Iquiri são as mais diferenciadas em relação aos outros grupos.

Em relação à hipótese da classificação das amostras a partir das disssimilaridades de composição química utilizando-se a análise de conglomerados, os resultados mostraram a formação de 6 grupos independentes, sendo que 3 deles são constituídos basicamente de amostras de cerâmicas das fases Iquiri, Xapuri e Quinari. Um dos outros grupos formados é constituído de uma mistura de amostras das fases Iaco e Jacuru. Dos outros dois grupos restante, um é contituído basicamente de amostras do Sítio Los Angeles e o outro de uma mistura do restante das amostras do Los Angeles com amostras das fases Iaco e 
Tabela 3. Caracterização dos grupos definidos em função da composição química

\begin{tabular}{|c|c|c|c|c|c|c|}
\hline & Grupo A & Grupo B & Grupo C & Grupo D & Grupo E & Grupo F \\
\hline Elemento & $M \pm \sigma$ & $\boldsymbol{M} \pm \sigma$ & $\boldsymbol{M} \pm \sigma$ & $M \pm \sigma$ & $M \pm \sigma$ & $\boldsymbol{M} \pm \sigma$ \\
\hline $\mathrm{Al}(\%)$ & $6,84 \pm 1,74$ & $8,25 \pm 2,44$ & $8,33 \pm 1,80$ & $8,60 \pm 1,69$ & $11,29 \pm 1,34$ & $10,09 \pm 1,59$ \\
\hline $\mathrm{Ce}(\mathrm{ppm})$ & $42,01 \pm 13,03$ & $48,48 \pm 19,35$ & $48,88 \pm 18,88$ & $72,03 \pm 7,65$ & $86,45 \pm 22,15$ & $68,11 \pm 12,45$ \\
\hline Co (ppm) & $7,27 \pm 1,25$ & $7,26 \pm 3,12$ & $8,62 \pm 4,07$ & $12,56 \pm 2,07$ & $25,41 \pm 6,86$ & $15,39 \pm 2,38$ \\
\hline $\mathrm{Cr}(\mathrm{ppm})$ & $55,47 \pm 8,52$ & $69,25 \pm 24,89$ & $73,96 \pm 24,15$ & $77,32 \pm 7,58$ & $100,27 \pm 9,93$ & $85,29 \pm 12,61$ \\
\hline $\mathrm{Cs}(\mathrm{ppm})$ & $14,62 \pm 3,94$ & $12,32 \pm 3,53$ & $14,52 \pm 2,73$ & $14,16 \pm 2,77$ & $14,09 \pm 4,27$ & $13,87 \pm 3,95$ \\
\hline Dy (ppm) & $3,75 \pm 0,52$ & $3,55 \pm 1,31$ & $3,36 \pm 0,95$ & $4,92 \pm 0,4$ & $7,26 \pm 2,59$ & $5,33 \pm 1,25$ \\
\hline $\mathrm{Eu}(\mathrm{ppm})$ & $0,90 \pm 0,3$ & $0,99 \pm 0,44$ & $0,94 \pm 0,40$ & $1,63 \pm 0,17$ & $2,43 \pm 1,03$ & $1,65 \pm 0,47$ \\
\hline $\mathrm{Fe}(\%)$ & $1,21 \pm 0,27$ & $3,43 \pm 1,69$ & $4,09 \pm 1,22$ & $3,63 \pm 0,45$ & $5,19 \pm 0,47$ & $4,46 \pm 0,64$ \\
\hline $\mathrm{Hf}(\mathrm{ppm})$ & $8,61 \pm 2,91$ & $5,84 \pm 1,97$ & $6,14 \pm 1,02$ & $7,36 \pm 0,81$ & $7,08 \pm 0,80$ & $6,83 \pm 1,19$ \\
\hline $\mathrm{Mn}(\mathrm{ppm})$ & $141,60 \pm 59,77$ & $180,77 \pm 129,24$ & $106,83 \pm 45,90$ & $225,64 \pm 87,57$ & $406,58 \pm 268,41$ & $383,85 \pm 141,62$ \\
\hline $\mathrm{Na}(\mathrm{ppm})$ & $519,28 \pm 116,39$ & $473,07 \pm 163,88$ & $1258,85 \pm 316,76$ & $1633,43 \pm 557,13$ & $745,75 \pm 263,74$ & $1173,54 \pm 558,2$ \\
\hline $\mathrm{Rb}(\mathrm{ppm})$ & $75,69 \pm 15,24$ & $88,87 \pm 22,78$ & $94,30 \pm 24,24$ & $116,84 \pm 14,16$ & $111,47 \pm 25,18$ & $100,15 \pm 18,44$ \\
\hline $\mathrm{Sb}(\mathrm{ppb})$ & $784,93 \pm 213,89$ & $657,87 \pm 202,31$ & $770,36 \pm 143,06$ & $756,3 \pm 132,21$ & $773,56 \pm 218,72$ & $758,32 \pm 201,97$ \\
\hline $\mathrm{Sc}(\mathrm{ppm})$ & $10,89 \pm 2,63$ & $12,72 \pm 4,27$ & $14,70 \pm 4,07$ & $16,33 \pm 1,17$ & $19,20 \pm 1,36$ & $17,25 \pm 2,21$ \\
\hline $\mathrm{Sm}(\mathrm{ppm})$ & $7,27 \pm 2,82$ & $7,49 \pm 3,94$ & $6,34 \pm 3,45$ & $9,55 \pm 1,64$ & $13,04 \pm 6,22$ & $9,85 \pm 2,51$ \\
\hline $\mathrm{Ta}(\mathrm{ppm})$ & $1,51 \pm 0,32$ & $1,21 \pm 0,38$ & $1,32 \pm 0,41$ & $1,59 \pm 0,16$ & $1,56 \pm 0,14$ & $1,47 \pm 0,20$ \\
\hline Th (ppm) & $11,59 \pm 2,21$ & $11,20 \pm 3,96$ & $11,87 \pm 3,41$ & $12,46 \pm 0,93$ & $15,53 \pm 1,14$ & $13,72 \pm 1,64$ \\
\hline $\mathrm{Ti}(\mathrm{ppm})$ & $3605,00 \pm 872,65$ & $3108,03 \pm 822,30$ & $3234,88 \pm 723,73$ & $4137,57 \pm 277,68$ & $4129,34 \pm 381,47$ & $3968,23 \pm 458,79$ \\
\hline$\underline{\mathrm{V}(\mathrm{ppm})}$ & $67,84 \pm 14,98$ & $90,75 \pm 32,85$ & $103,13 \pm 24,53$ & $98,46 \pm 13,42$ & $133,65 \pm 18,27$ & $121,82 \pm 22,28$ \\
\hline
\end{tabular}

Jacuru, além de algumas poucas amostras das fases Xapuri e Quinari.

A dispersão das amostras das fases Iaco e Jacuru, assim como também a definição dos outros grupos pelo método de conglomerados foi concordante com os resultados obtidos pela análise de discriminantes. Pode-se então concluir que as fases arqueologicamente mais bem definidas são as fases Iquiri, Xapuri e Quinari e que através dos estudos realizados pode-se verificar que a metodologia de classificação empregada pelos arqueólogos reproduz bons resultados. Sugere-se porém, a partir da classificação feita pela composição química, que este seja "ajustado" em busca de microdiferenciações que possam explicar a reclassificação de alguns dos fragmentos cerâmicos em função da composição química.

\section{AGRADECIMENTOS}

Ao $\mathrm{CNPq}$ pelo suporte financeiro, ao IEN/CNEN pelo nitrogênio líquido e ao Dr. Luís Fernando Bellido pelas sugestões e correções.

\section{REFERÊNCIAS}

1. Franklin, U.M.; Vitali, V.; Archaeometry 1985, 27, 3.

2. Travesi, A.; Analisis por Activacion Neutronica: Teoria, prática y aplicaciones, Junta de Energia Nuclear; Madri, 1975; p.447.

3. De Soete, D.; Gijbels, R.; Hoste, J.; Neutron Actvation Analysis, John Wiley and Sons, Londres, 1972, p.177.

4. Dams, R.; J. Anal. Chem. 1990, 337, 492.
5. Perlman, I.; Asaro, F.; Archaeometry 1969, 11, 21.

6. Harbottle, G.; Radiochemistry, The Chemical Society; Londres; 1976; p.33.

7. Hancock, R.G.V.; Archaeometry 1985, 27, 94.

8. Meggers, B.; Boletim do Museu Paranaense Emílio Goeldi, Série Antropologia 1990, 6, 183

9. Dias, O.; Carvalho, E.; Arqueo-IAB 1988, 14.

10. Latini, R. M.; Tese de Doutorado; UFF, Niterói, RJ, 1998.

11. Moon, P.; Rodrigues, A.; Istoé 2000, 1604, 92.

12. Dias, O.; Carvalho, E.; Aspectos da Arqueologia Amazônica 1981, 2, 21.

13. Bellido, A.V.B.; Tese de Doutorado; University of Manchester, Manchester, 1989.

14. Bellido, L.F.B.; Publicação IEN-67, 1995.

15. Gilmore, G.R.; Technical report Universities Research Reactor, 1983.

16. Bussad, W. O.; Miazaki, E. S.; Andrade, D. F.; Introdução à Análise de Agrupamentos; Associação Brasileira de Estatística; São Paulo, 1990.

17. Cooley, W. W.; Lohnes, P. R.; Multivariate Data Analysis; John Wiley and Sons; New York; 1971.

18. Valentin, J. L.; Ecologia Numérica: Uma Introdução à Análise Multivariada de Dados Ecológicos; Interciência; Rio de Janeiro; 2000.

19. Mardia, K. V.; Kent, J. T.; Birby, J. M.; Multivariate Analysis; Academic Press; Londres; 1979.

20. Mommsen, H.; Kreuser, A.; Weber, J.; Archaeology 1988, $30,47$.

21. Mahalanobis, P.C.; Proceedings of the National Institute of Science 1936, 2, 49. 